Gefässchirurgie 2015 · 20:127-134

DOI 10.1007/s00772-015-0012-y

Online publiziert: 18. März 2015

(c) Die Autor(en) 2015. Dieser Artikel ist auf

Springerlink.com mit Open Access verfügbar
Die primäre Varikose ist eine Volkskrankheit, die aufgrund der demografischen Entwicklung in Zukunft weiter an Bedeutung gewinnen wird. Im Spektrum der therapeutischen Interventionen bei vorliegender Stammveneninsuffizienz ist die klassische Operation mit Krossektomie und Stripping $(C / S)$ in Deutschland nach wie vor führend. Seit nunmehr 15 Jahren werden zunehmend endovenöse Techniken implementiert und der konventionellen Chirurgie im Rahmen von Studien wie auch in der täglichen Praxis gegenübergestellt. Insbesondere in den USA und in Großbritannien werten aktuelle Leitlinien die thermoablativen Verfahren bereits als Therapie der ersten Wahl $[22,28]$.

Im Vordergrund dieser neuen Behandlungsverfahren stehen die endovenöse Lasertherapie (ELT) und die Radiofrequenzablation (RFA). Die stärkste Evidenz durch umfangreiche randomisierte kontrollierte Studien (RCTs) liegt derzeit für die ELT vor [27], die daher im Fokus dieser systematischen Übersichtsarbeit steht. Zunächst soll ein Überblick über die klinische und sozioökonomische Relevanz der Varikose und das vorhandene therapeutische Repertoire gegeben werden. Ein kurzer Exkurs befasst sich mit dem wichtigen Aspekt der Rezidivprophylaxe im Rahmen der klassischen Operation. Um die Empfehlungen der US-amerikanischen und britischen Leitlinien besser einordnen zu können, wird abschließend die aktuelle Studienlage zur ELT dargestellt und ihr Stellenwert im Vergleich zur klassischen Operation diskutiert.

K. Rass

Zentrum für Venen und Haut, Simmerath, Detuschland

\title{
Ist die endovenöse Lasertherapie wirklich besser als die Stripping-Operation?
}

\author{
Epidemiologie, Ätiopathogenese \\ und klinische Relevanz \\ der primären Varikose
}

Die chronisch-venöse Funktionsstörung bzw. Insuffizienz (CVI) der Beinvenen zählt mit einer Prävalenz von 30-40\% der erwachsenen Bevölkerung zu den häufigsten Krankheitsbildern in westeuropäischen Ländern [34]. Die primäre Varikose stellt den Hauptanteil der Venenerkrankungen dar. Neben den sich über die Jahre entwickelnden Stauungssymptomen und der kosmetischen Beeinträchtigung, die für die Betroffenen eine Einschränkung ihrer Lebensqualität bedeuten, sind die potenziellen Komplikationen der Varikose von medizinischer und sozioökonomischer Bedeutung: tiefe Beinvenenthrombose, Lungenembolie, postthrombotisches Syndrom, Ulcus cruris venosum, arthrogenes Stauungssyndrom, sekundäres Lymphödem, erhöhte Suszeptibilität für Hautinfektionen (z. B. Dermatophytosen, Erysipel) und epidermale Sensibilisierungen (Typ-IV-Allergie) (Übersicht in [16]). Daten zur aktuellen Prävalenz des venösen Ulcus cruris in Deutschland reichen von etwa 80.000 (Raum Bonn, extrapoliert auf Deutschland) bis zu 1 Mio. betroffenen Patienten [14, 34]. Die jährliche Inzidenz thrombembolischer Ereignisse in Deutschland wird mit 150-200/100.000 Einwohner angegeben, ca. 40.000 Menschen versterben jährlich an den Folgen einer Lungenarterienembolie [42].

Die Ätiologie der primären Varikose ist vermutlich multifaktoriell. Angenommen werden genetisch determinierte strukturelle Veränderungen der Venenwand bzw. der Venenklappen, die $\mathrm{zu}$ einer Dilatation und Funktionsbeein- trächtigung mit pathologischen Refluxen im epifaszialen Venensystem führen [24].

) Die Ätiologie der primären Varikose ist multifaktoriell

Pathogenetisch bedeutsam scheint eine Remodellierung der Venenwand durch Aktivierung bestimmter Matrixmetalloproteinasen zu sein. In der aktuellen Literatur finden sich vermehrt Hinweise dafür, dass dieses „Remodelling“ allein durch einen chronisch bestehenden erhöhten venösen Füllungsdruck, wie z. B. durch lange Steh- oder Sitzbelastung, Bewegungsmangel und Adipositas als typische Realisationsfaktoren der primären Varikose, induziert werden kann [30]. Die Folgen sind u. a. venöse Hypertension, kapilläre Umbauprozesse (Ausbildung von Fibrinmanschetten, Diffusionsbarriere für Sauerstoff), sowie eine durch Leukozyten unterhaltene chronische Gewebsentzündung als Ausdruck der CVI (Übersicht in [35]).

\section{Therapeutisches Spektrum}

Eine kausale Therapie der primären Varikose, d. h. eine Rekonstitution der insuffizienten Venenabschnitte und Maßnahmen zur langfristigen Verhinderung des venösen „Remodelling“, steht derzeit nicht zur Verfügung. Im Sinne einer symptomorientierten Therapie und zur Prävention der Entwicklung einer CVI und der oben genannten sekundären Komplikationen kommen unterschiedliche konservative und invasive Verfahren zum Einsatz. Die invasiven Therapien zielen auf eine Beseitigung des venösen Reflu- 
xes, entweder durch Entfernung der refluxiven Venen im Rahmen der konventionellen Varizenchirurgie oder durch endovenöse Ablation.

\section{Krossektomie und Stripping (C/S)}

Bis vor etwa 10 Jahren galt die Operation mit $\mathrm{C} / \mathrm{S}$ als alleinige Standardtherapie der behandlungsbedürftigen Stammvarikose. Die Operationstechnik geht insbesondere auf Arbeiten von Babcock und Homans aus den ersten zwei Jahrzehnten des vergangenen Jahrhunderts zurück und umfasst einerseits die niveaugleiche bzw. mündungsnahe Ligatur an der saphenofemoralen bzw. saphenopoplitealen Junktion nach Absetzen aller Krossenseitenäste (Krossektomie) und andererseits das Stripping der insuffizienten Stammvene mit unterschiedlichen Sonden. Systematische Untersuchungen zur Effektivität der Varizenoperation zeigten, dass die Operationsmethode zwar mit einem günstigen Komplikationsprofil, jedoch mit einer relevanten Rezidivquote behaftet ist $[17,20]$. Aktuelle randomisierte Studien weisen duplexsonographisch nachweisbare inguinale Rezidive 5 Jahre postoperativ mit einer Häufigkeit von bis zu 46\% auf [41]. Eine klinische Relevanz mit entsprechend ausgeprägter Rezidivvarikose und bestehender Indikation für eine chirurgische Revision eines inguinalen oder poplitealen Krossenrezidivs wird 5 Jahre postoperativ bei ca. $10 \%$ der Patienten beobachtet [17].

\section{Neovaskularisation und daraus resultierende Strategien}

Als ursächlich für das Auftreten von inguinalen Krossenrezidiven gelten einerseits Operationsfehler mit lang belassenen Krossenstümpfen, die in über 70\% der Fälle beobachtet werden [25]. Bei korrekter Operationstechnik wird die Angiogenese aus dem Gebiet der ehemaligen saphenofemoralen Junktion mit Anschluss an präexistente Venen (z. B. V. saphena accessoria anterior) als führende Rezidivursache angesehen [3]. In diesem Prozess kommt vermutlich dem freiliegenden Stumpfendothel eine wichtige Bedeutung zu.

Aus der Kenntnis des Phänomens der Neovaskularisation heraus wurden bereits diverse Strategien entwickelt. So zie- len Barrieretechniken darauf ab, die von der saphenofemoralen Mündung ausgehende Neoangiogenese mechanisch zu inhibieren. Die invaginierende Endothelnaht des ligierten Krossenstumpfes führt zu einer Reduktion duplexsonographisch nachweisbarer inguinaler Rezidive von $>70 \%$ [21]. Mittels Silikonimplantat wurde 5 Jahre nach durchgeführter inguinaler Rekrossektomie eine Reduktion der Neovaskularisationsrate von 45 auf 9\% beobachtet [8].

\section{》) Barrieremaßnahmen sind ein unerlässlicher Bestandteil der Krossektomie}

In einer randomisierten kontrollierten Studie zum Vergleich der inguinalen Krossektomie mit oder ohne Polytetrafluorethylen-Patch zeigte sich ebenfalls ein deutlicher Nutzen für die Barrieretechnik mit Reduktion der Neovaskularisationsrate von 46 auf $23 \% 3$ Jahre postoperativ [41]. Die Naht der Fossa ovalis als Barrieremaßnahme scheint bei Verzicht auf das Einbringen von allogenem Material eine vergleichbare Effektivität bei geringerem Komplikationspotenzial aufzuweisen [10]. Aufgrund dieser Studienergebnisse sind Barrieremaßnahmen heute sicher als unerlässlicher Bestandteil der Krossektomie einzustufen.

\section{Interventionelle Therapie der primären Varikose: endovenöse Lasertherapie (ELT)}

Ungeachtet der Problematik der Neovaskularisation zeichnet sich seit der Jahrtausendwende mit der Etablierung interventioneller Therapien insbesondere im Bereich der Kardiologie und der arteriellen Gefäßchirurgie ein Paradigmenwechsel auch in der Phlebologie ab: Anforderungen an eine moderne Varizentherapie sollten minimale Invasivität, das Ausbleiben insbesondere schwerwiegender Komplikationen, das Erreichen eines ästhetisch optimalen Ergebnisses sowie die schnelle Rekonvaleszenz des Patienten unter geringer Beeinträchtigung der Lebensqualität sein. $\mathrm{Zu}$ den bei der Varikose angewandten Techniken zählen die thermoablativen Verfahren der ELT, der RFA und der Heißdampfapplikation sowie die nicht thermischen Verfahren der Schaumsklerotherapie, der mechanochemischen $\mathrm{Ab}$ lation und der Verklebung mit Acrylatklebern.

Erste systematische Untersuchungen zur ELT gehen auf Boné, Navarro und Min zurück und wurden 2001 erstmals publiziert [26]. Für die mit einfachen Glasfasern ("bare fibers") arbeitende 1. Generation der ELT kommen Diodenlaser der Wellenlängen 810, 940 und $980 \mathrm{~nm}$ zum Einsatz, wobei relevante Unterschiede des Wirkmechanismus und der Effektivität nicht anzunehmen sind. Die intravasale Laseranwendung des oben genannten Spektrums führt zu einer Erhitzung des Blutes über die Energieabsorption durch Hämoglobin, zur Ausbildung von Dampfblasen und sekundär zu einer thermischen Gefäßwandschädigung mit konsekutiver Thrombusbildung [31]. Im Gegensatz zur RFA sind punkt- und schlitzförmige Perforationen der Venenwand bei den Lasern der 1. Generation häufig und die Gefäßkontraktion ist wesentlich geringer ausgeprägt [46]. Bei erfolgreicher Ablation ist im späteren Verlauf eine Gefäßschrumpfung und letztlich ein fibrotischer Abbau der behandelten Vene zu beobachten.

Die Laserfaser wird in Seldinger-Technik intravenös eingebracht, ultraschallgesteuert in der Krosse positioniert und nach Applikation der Tumeszenzlokalanästhesie kontinuierlich oder im Impulsbetrieb unter entsprechender Energieabgabe zurückgezogen. Zur Erzielung einer optimalen Verschlussrate sollten Energiedosen von mindestens $60 \mathrm{~J} / \mathrm{cm}$ Venenlänge eingebracht werden [44].

Ein wesentlicher Nachteil der ELT der 1. Generation besteht, wie bereits mehrere RCTs zeigen konnten, darin, dass trotz der propagierten minimalen Invasivität die Komplikationsraten (Schmerzen, Hämatome, phlebitische Reaktionen, Dyspigmentierungen) größer sind und auch die postoperative Lebensqualität und Rekonvaleszenz im Vergleich insbesondere zu den RFA-Verfahren stärker beeinträchtigt werden $[1,23,29,38,43]$. Vermutlich sind die eingesetzten Wellenlängen mit dem oben genannten Wirkmechanismus, vor allem aber die axiale, punktförmige Abstrahlung des Laserlichts mit auftretenden Gefäßwandper- 
forationen hierfür verantwortlich. Daher wurden ab 2005 zunächst Laser mit größerer Wellenlänge (1320 nm Nd:YAG, $1470 \mathrm{~nm}$ Diodenlaser) zur endothermischen Ablation eingeführt, die ein höheres Absorptionsmaximum in wasserhaltigem Gewebe, i. e. der Venenwand, aufweisen. Ob diese Strategie bei Einsatz vergleichbarer Energiedosen tatsächlich zu geringeren Nebenwirkungen führt, ist in der Literatur allerdings uneindeutig [32, 38]. Ein weiterer technischer Fortschritt der ELT wurde mit der Änderung der Abstrahlungsgeometrie des Laserlichts erreicht. 2008 wurde die Radialfaser etabliert, die mit einem 1470 nm Diodenlaser betrieben wird und das Laserlicht in einem Winkel von $90^{\circ}$ ringförmig auf das Endothel projiziert. Der Radiallaser verursacht bei Einsatz gleicher Leistungen und vergleichbarer Energiedosen signifikant geringere Schmerzen als die axiale Faser des Diodenlasers der 1. Generation [15]. Vergleichende Untersuchungen mit Krossektomie und Stripping liegen für den Radiallaser derzeit nicht vor.

\section{Vergleich von $\mathrm{C} / \mathrm{S}$ und ELT}

C/S auf der einen Seite und endovenöse Ablation auf der anderen unterscheiden sich im Hinblick auf das Therapieergebnis vor allem in der postoperativen anatomischen und hämodynamischen Situation an der Krossenmündung. Während der venöse Blutabfluss über die Krossen nach leitliniengerechter Operation vollständig unterbunden ist und die Notwendigkeit dieses Vorgehens galt bislang als Paradigma -, bleibt nach Stammvenenablation insbesondere der V. saphena magna (VSM) eine Perfusion der Krosse über die Seitenäste des Venensterns regelhaft erhalten. Dies führt zu unterschiedlichen Mustern auftretender Rezidive. Während nach korrekter Krossektomie Rezidive hauptsächlich durch Neovaskularisation entstehen, sind Rekanalisierungen ablatierter Venen und die Ausbildung von postoperativen Refluxen über zuvor suffiziente Krossenseitenäste zu beobachtende Phänomene nach endovenöser Therapie, die zu einem klinisch relevanten Rezidiv führen können [45].

Größere evidenzbasierte Untersuchungen im Sinne von RCTs sind weit

Gefässchirurgie 2015 - 20:127-134 DOI 10.1007/s00772-015-0012-y

○ Die Autor(en) 2015. Dieser Artikel ist auf Springerlink.com mit Open Access verfügbar

\section{K. Rass \\ Ist die endovenöse Lasertherapie wirklich besser als die Stripping-Operation?}

\section{Zusammenfassung}

Hintergrund. Im Spektrum der therapeutischen Interventionen bei primärer Stammveneninsuffizienz ist in Deutschland die klassische Operation mit Krossektomie und Stripping die häufigste Behandlungsform. Seit 15 Jahren werden zunehmend minimal-invasive endovenöse Techniken eingesetzt und der konventionellen Chirurgie gegenübergestellt. In den USA und in Großbritannien (NICE) bewerten aktuelle Leitlinien die thermoablativen Katheterverfahren bereits als Therapie der ersten Wahl.

Ziel der Arbeit. Darstellung der aktuellen Evidenz zur endovenösen Lasertherapie (ELT) im Vergleich zur konventionellen Strippingoperation (C/S) auf der Basis randomisierter kontrollierter Studien. Bewertung von Sicherheit, Nebenwirkungen, Lebensqualität und langfristiger Effektivität beider Verfahren. Material und Methoden. Selektive Literaturanalyse auf der Basis einer PubMed-Recherche mit dem Schwerpunkt auf randomisierte kontrollierte Studien (RCT) zum Vergleich von ELT und C/S.
Ergebnisse. Es liegen 10 Studienkollektive aus RCTs mit insgesamt 2210 eingeschlossenen Patienten vor. Im Hinblick auf Sicherheit, Nebenwirkungen, Besserung von Symptomen, der Lebensqualität und Patientenzufriedenheit sowie der mittelfristigen klinischen Rezidivrate besteht eine Gleichwertigkeit von ELT und C/S. Ein Vorteil für die ELT besteht in der schnelleren Rekonvaleszenz, ein Nachteil in den häufiger zu beobachtenden duplexsonographischen Rezidiven, die als Surrogatparameter für langfristige klinisch relevante Rezidive eingestuft werden.

Schlussfolgerungen. Zum gegenwärtigen Zeitpunkt liegt keine Evidenz vor, die es begründen würde, ELT gegenüber $C / S$ zu favorisieren.

\section{Schlüsselwörter}

Varikose $\cdot$ Krossektomie und Stripping .

Endovenöse Lasertherapie .

Randomisierte kontrollierte Studien . Leitlinien

\section{Is endovenous laser ablation really superior to conventional stripping?}

\section{Abstract}

Background. Conventional surgery with high ligation and stripping is the most common therapeutic intervention for primary insufficiency of saphenous veins in Germany. Over the last 15 years minimally invasive endovenous approaches have increased in popularity and compete against conventional surgery. In current clinical guidelines from the USA and the National Institute for Health and Care Excellence of the UK, thermal ablative catheter techniques are already rated as the treatment of first choice.

Objectives. Demonstration of the current evidence from randomized controlled trials (RCT) for endovenous laser treatment (ELT) compared with conventional stripping surgery (C/S). Assessment of safety, side effects, quality of life and long-term efficacy of both procedures.

Material and methods. Selective analysis of the literature based on a PubMed search fo- cusing on RCT for the comparison of ELT and $\mathrm{C} / \mathrm{S}$ (high ligation with stripping). Results. A total of 10 study collectives from RCT comprising 2210 patients could be identified. In terms of safety, side effects, improvement of symptoms, quality of life and patient satisfaction, as well as mid-term clinical efficacy, ELT and C/S are on a par. Compared with $\mathrm{C} / \mathrm{S}$, ELT has an advantage concerning convalescence but a disadvantage regarding duplex detected recurrence, which is a predictor for long-term clinical recurrence.

Conclusion. To date no evidence is available that would justify ELT to be recommended over $\mathrm{C} / \mathrm{S}$.

\section{Keywords}

Varicose veins - High ligation and stripping . Endovenous laser ablation .

Randomized controlled trials · Guidelines überwiegend zur ELT der 1. Generation im Vergleich mit C/S der VSM verfügbar [27]. Die aktuelle Datenlage soll im
Folgenden eingehend dargestellt werden. 


\section{Übersichten}

\begin{tabular}{|c|c|c|c|c|c|c|c|c|c|c|c|}
\hline Erstautor & Publiziert & $\begin{array}{l}\text { Stichprobe } \\
\text { ITT- } \\
\text { Population }\end{array}$ & Vergleich & $\begin{array}{c}\text { Prozeduren } \\
\text { ELT / C/S }\end{array}$ & ELT-Dosis & $\begin{array}{l}\text { FU- } \\
\text { Dauer }\end{array}$ & $\begin{array}{l}\text { FU- } \\
\text { Rate }\end{array}$ & $\begin{array}{l}\text { Okklusion } \\
\text { (ELT) }\end{array}$ & $\begin{array}{c}\text { OP-Erfolg } \\
\text { (C/S) }\end{array}$ & $\begin{array}{l}\text { Duplex- } \\
\text { Krossenresidiv } \\
\text { ELT / C/S }\end{array}$ & $\begin{array}{l}\text { Klinisches } \\
\text { Rezidiv } \\
\text { ELT / C/S }\end{array}$ \\
\hline $\begin{array}{l}\text { Rasmussen } \\
{[36,37,39]}\end{array}$ & $\begin{array}{l}\text { Aug. } 07 \\
\text { Jan. } 10 \\
\text { Aug. } 13\end{array}$ & $\begin{array}{l}\text { 121 Patienten } \\
137 \text { Beine }\end{array}$ & $\begin{array}{c}\text { ELT } 12 \mathrm{~W} 1,5 \mathrm{~s} \text { Impuls } \\
\mathrm{C} / \mathrm{S}\end{array}$ & $69 / 68$ & $73,5 \mathrm{~J} / \mathrm{cm}$ & $\begin{array}{l}6 \mathrm{M} . \\
2 \mathrm{~J} . \\
5 \mathrm{~J} .\end{array}$ & $\begin{array}{l}76 \% \\
90 \% \\
67 \%\end{array}$ & $100,0 \%$ & $97,1 \%$ & \begin{tabular}{|c|}
$5,6 \% / 2,0 \%$ \\
$16,9 \% / 13,8 \%$ \\
$?$
\end{tabular} & \begin{tabular}{|c|} 
nb \\
$27,7 \% / 43,1 \% * *$ \\
$46,6 \% / 54,6 \% * *$
\end{tabular} \\
\hline $\begin{array}{l}\text { Disselhoff } \\
\text { [11-13] }\end{array}$ & $\begin{array}{l}\text { Juni } 08 \\
\text { Dez. } 08 \\
\text { Aug. } 11 \\
\end{array}$ & $\begin{array}{l}120 \text { Patienten } \\
120 \text { Beine }\end{array}$ & $\begin{array}{c}\text { ELT } 810 \mathrm{~nm} 14 \mathrm{~W} \\
\mathrm{C} / \mathrm{cS}\end{array}$ & $60 / 60$ & $57 \mathrm{~J} / \mathrm{cm}$ & $\begin{array}{l}2 \mathrm{~J} . \\
5 \mathrm{~J} .\end{array}$ & $\begin{array}{l}93 \% \\
63 \%\end{array}$ & $95,0 \%$ & $100 \%$ & $\begin{array}{c}16,1 \% / 20,0 \%^{*} \\
22 \% / 28 \%^{*}\end{array}$ & $\begin{array}{l}23 \% / 34 \% \\
38 \% / 49 \%\end{array}$ \\
\hline $\begin{array}{l}\text { Darwood } \\
\text { [7] }\end{array}$ & März 08 & $\begin{array}{l}118 \text { Patienten } \\
136 \text { Beine }\end{array}$ & $\begin{array}{c}\text { ELT } 810 \mathrm{~nm} 12 \mathrm{~W} 1 \mathrm{~s} \text { Impuls } \\
\text { ELT } 810 \mathrm{~nm} 14 \mathrm{~W} \mathrm{cW} \\
\mathrm{C} / \mathrm{S}\end{array}$ & $80 / 34$ & $\begin{array}{c}60,6 \mathrm{~J} / \mathrm{cm} \\
71,1 \mathrm{~J} / \mathrm{cm} \\
-\end{array}$ & $3 \mathrm{M}$ & $90 \%$ & $\begin{array}{l}97,6 \% ! \\
89,7 \%\end{array}$ & $87,5 \%^{*}$ & $7,0 \% / 3,1 \%$ & $\mathrm{nb}$ \\
\hline $\begin{array}{c}\text { Pronk } \\
{[33]}\end{array}$ & Nov. 10 & $\begin{array}{c}122 \text { Patienten } \\
130 \text { Beine }\end{array}$ & $\begin{array}{c}\text { ELT } 980 \mathrm{~nm} 12 \mathrm{~W} \mathrm{cW} \\
\mathrm{C} / \mathrm{S}\end{array}$ & $62 / 68$ & $64,5 \mathrm{~J} / \mathrm{cm}$ & $1 \mathrm{~J}$. & $98 \%$ & $\mathrm{nb}$ & $\mathrm{nb}$ & $10 \% / 9 \%$ & $6 \% / 5,4 \%$ \\
\hline $\begin{array}{l}\text { Christenson } \\
{[6]}\end{array}$ & Nov. 10 & 204 Beine & $\begin{array}{c}\text { ELT } 980 \mathrm{~nm} \mathrm{10-12} \mathrm{W} 1,5 \mathrm{~s} \text { Impuls } \\
\mathrm{C} / \mathrm{S}\end{array}$ & $100 / 100$ & $75,9 \mathrm{~J} / \mathrm{cm}$ & $2 \mathrm{~J}$. & $97 \%$ & $99,0 \%$ & $100,0 \%$ & $\begin{array}{l}7,1 \% / 0 \% * \\
\text { ITT } p=0,016\end{array}$ & $\mathrm{nb}$ \\
\hline $\begin{array}{c}\text { Carradice } \\
{[4,5]}\end{array}$ & $\begin{array}{l}\text { Apr. } 11 \\
\text { Aug.11 }\end{array}$ & \begin{tabular}{|c|}
280 Patienten \\
280 Beine \\
\end{tabular} & $\begin{array}{c}\text { ELT } 810 \mathrm{~nm} 14 \mathrm{~W} \mathrm{cW} \\
\mathrm{C} / \mathrm{S}\end{array}$ & $139 / 137$ & $95 \mathrm{~J} / \mathrm{cm}$ & $1 \mathrm{~J}$. & $85 \%$ & $99,3 \%$ & $92,4 \%$ & $? / 15 \%$ & $4 \% / 20,4 \%$ \\
\hline $\begin{array}{c}\text { Rasmussen } \\
{[38]}\end{array}$ & Aug. 11 & $\begin{array}{l}250 \text { Patienten } \\
287 \text { Beine }\end{array}$ & $\begin{array}{c}\text { ELT } 980 \mathrm{~nm} \text { und } 1470 \mathrm{~nm} \text { pw oder } \mathrm{cw} \\
\mathrm{C} / \mathrm{S} \\
(+2 \text { Arme: RFA, UGFS, weitere } 250 \mathrm{P})\end{array}$ & $144 / 142$ & $76,5 \mathrm{~J} / \mathrm{cm}$ & $1 \mathrm{~J}$. & $80 \%$ & $100,0 \%$ & $97,2 \%$ & $5,8 \% / 4,8 \%^{*}$ & $11,6 \% / 14,8 \% * *$ \\
\hline $\begin{array}{l}\text { Rass } \\
{[40]}\end{array}$ & Jan. 12 & $\begin{array}{l}400 \text { Patienten } \\
400 \text { Beine }\end{array}$ & $\begin{array}{c}\mathrm{ELT} 810 \mathrm{~nm} 20 \mathrm{~W} \mathrm{cW} \\
\mathrm{C} / \mathrm{S}\end{array}$ & $185 / 161$ & $\begin{array}{l}22,5 \mathrm{~J} / \mathrm{cm}^{2} \\
48,7 \mathrm{~J} / \mathrm{cm}\end{array}$ & $2 \mathrm{~J}$. & $91 \%$ & $98,9 \%$ & $100,0 \%$ & $17,8 \% / 1,3 \% * *$ & $16,2 \% / 23,1 \%^{*}$ \\
\hline $\begin{array}{c}\text { Flessenkämper } \\
{[18,19]}\end{array}$ & $\begin{array}{l}\text { Febr. } 13 \\
\text { Mai } 14\end{array}$ & 449 Patienten & $\begin{array}{c}\text { ELT } 980 \mathrm{~nm} 30 \mathrm{~W} \mathrm{cW} \\
\mathrm{C} / \mathrm{S} \\
\mathrm{C}+\mathrm{ELT}\end{array}$ & $142 / 159$ & $85,4 \mathrm{~J} / \mathrm{cm}$ & $\begin{array}{l}2 \mathrm{M} \\
6 \mathrm{M} \\
2 \mathrm{~J}\end{array}$ & $\begin{array}{l}100 \% \\
86 \% \\
74 \%\end{array}$ & $98,6 \%$ & $100,0 \%$ & $\begin{array}{c}26,8 \% / 0 \%^{*} \\
31,5 \% / 1,6 \%{ }^{*} \\
\text { keine } \% \\
\text { Angaben }\end{array}$ & $\begin{array}{c}\text { nb } \\
7,9 \% / 9,4 \% * * \\
\text { keine } \% \\
\text { Angaben }\end{array}$ \\
\hline $\begin{array}{l}\text { Biemans } \\
\text { [2] }\end{array}$ & Sep. 13 & $\begin{array}{l}146 \text { Patienten } \\
160 \text { Beine }\end{array}$ & $\begin{array}{c}\text { ELT } 940 \mathrm{~nm} \text { ?W cw } \\
\text { C/S } \\
\text { (+1 Arm: UGFS, weitere } 80 \text { Beine) }\end{array}$ & $76 / 65$ & $59,2 \mathrm{~J} / \mathrm{cm}$ & $1 \mathrm{~J}$. & $100 \%$ & $\mathrm{nb}$ & $\mathrm{nb}$ & $11,5 \% / 11,8 \%^{*}$ & $\mathrm{nb}$ \\
\hline
\end{tabular}

Abb. 1 \ Randomisierte Studien zum Vergleich der endovenösen Lasertherapie (ELT) mit Krossektomie/Stripping (C/S) - Studiendesign und Effektivität. Rote Felder signifikanter Vorteil für ELT, blaue Felder signifikanter Vorteil für C/S, graue Felder kein signifikanter Unterschied. *primäre Studienvariable ** sekundäre Studienvariable. ITT Intention to treat, ELT endovenöse Lasertherapie, FU Follow-up, cS Cryostripping, RFA Radiofrequenzablation, UGFS "ultrasound guided foam sclerotherapy", $M$. Monate, J. Jahre, $n b$ nicht berichtet, Krossektomie/Stripping (C/S)

\begin{tabular}{|c|c|c|c|c|c|c|c|c|c|c|c|}
\hline Erstautor & Infektion & $\begin{array}{c}\text { Postop. } \\
\text { Schmerzen }\end{array}$ & $\begin{array}{c}\text { Hämatome } \\
\text { Blutungen }\end{array}$ & Induration & $\begin{array}{c}\text { Par- } \\
\text { ästhesie }\end{array}$ & Phlebitis & $\begin{array}{l}\text { Pigmen- } \\
\text { tierung }\end{array}$ & $\begin{array}{c}\text { Rekon- } \\
\text { valeszenz }\end{array}$ & $\begin{array}{l}\text { Venen- } \\
\text { Scoring }\end{array}$ & $\begin{array}{l}\text { Lebens- } \\
\text { qualität }\end{array}$ & $\begin{array}{c}\text { Patienten- } \\
\text { zufriedenheit }\end{array}$ \\
\hline $\begin{array}{c}\text { Rasmussen } \\
{[36,37,39]}\end{array}$ & & SF-36 BP** & & $\mathrm{nb}$ & & & $\mathrm{nb}$ & $*$ & VCSS** & $\begin{array}{l}\text { AVVQ** } \\
\text { SF }-36^{* *}\end{array}$ & $\mathrm{nb}$ \\
\hline $\begin{array}{l}\text { Disselhoff } \\
\text { [11-13] }\end{array}$ & & VAS & & & & & $\mathrm{nb}$ & & VCSS* & $A W Q^{*}$ & $\mathrm{nb}$ \\
\hline $\begin{array}{c}\text { Darwood } \\
\text { [7] }\end{array}$ & & & & $\mathrm{nb}$ & & & $\mathrm{nb}$ & & VCSS & $A \cup Q^{*}$ & \\
\hline $\begin{array}{c}\text { Pronk } \\
\text { [33] }\end{array}$ & $*$ & VAS** & $*$ & $\mathrm{nb}$ & $*$ & $\mathrm{nb}$ & $\mathrm{nb}$ & & $\mathrm{nb}$ & EuroQol-5D** & \\
\hline $\begin{array}{c}\text { Christenson } \\
{[6]}\end{array}$ & * & * & * & $\mathrm{nb}$ & * & * & $\mathrm{nb}$ & $*$ & VCss* & $\begin{array}{l}\text { AVVQ } \\
\text { SF-36** }\end{array}$ & $\mathrm{nb}$ \\
\hline \multirow{2}{*}{$\begin{array}{c}\text { Carradice } \\
{[4,5]}\end{array}$} & & \multirow{2}{*}{$\begin{array}{l}\text { VAS } \\
\text { SF-36* }\end{array}$} & & \multirow{2}{*}{$\mathrm{nb}$} & & & & \multirow{2}{*}{$*$} & \multirow{2}{*}{ VCSS $^{* *}$} & \multirow{2}{*}{$\begin{array}{c}\text { AVVQ** } \\
\text { EuroQol-5D** }\end{array}$} & kosm. Resultat* \\
\hline & & & & & & & & & & & Gesamtresultat* \\
\hline $\begin{array}{c}\text { Rasmussen } \\
{[38]}\end{array}$ & & $\begin{array}{l}\text { VAS } \\
\text { SF-36** }\end{array}$ & & $\mathrm{nb}$ & & & $\mathrm{nb}$ & $*$ & VCSS** & $\begin{array}{l}\text { AVVQ } \\
\text { SF-36** }\end{array}$ & $\mathrm{nb}$ \\
\hline \multirow{2}{*}{$\begin{array}{c}\text { Rass } \\
{[40]}\end{array}$} & \multirow{2}{*}{$*$} & \multirow{2}{*}{ VAS** } & \multirow{2}{*}{$*$} & \multirow{2}{*}{$*$} & \multirow{2}{*}{$*$} & \multirow{2}{*}{$* *$} & \multirow{2}{*}{$*$} & \multirow{2}{*}{$*$} & \multirow{2}{*}{ HVVSS** } & \multirow{2}{*}{ CIVIQ2** } & kosm. Resultat** \\
\hline & & & & & & & & & & & Gesamtresultat** \\
\hline $\begin{array}{c}\text { Flessenkămper } \\
{[18,19]}\end{array}$ & $\mathrm{nb}$ & VAS $^{* *}$ & $*$ & $\mathrm{nb}$ & $* *$ & $\mathrm{nb}$ & & & $\begin{array}{l}\text { VCSS I } \\
\text { VDS** }\end{array}$ & $\mathrm{nb}$ & $\mathrm{nb}$ \\
\hline $\begin{array}{c}\text { Biemans } \\
{[2]}\end{array}$ & $*$ & $\mathrm{nb}$ & $\mathrm{nb}$ & $\mathrm{nb}$ & $* *$ & $* *$ & $*$ & $\mathrm{nb}$ & CEAP** & $\begin{array}{c}\text { CIVIQ } \\
\text { EuroQol-5D** }\end{array}$ & $\mathrm{nb}$ \\
\hline
\end{tabular}

Abb. $2 \Delta$ Randomisierte Studien zum Vergleich der endovenösen Lasertherapie (ELT) mit Krossektomie/Stripping (C/S) Nebenwirkungen, Rekonvaleszenz, Venen-Scoring und Lebensqualität. Rote Felder signifikanter Vorteil für ELT, blaue Felder signifikanter Vorteil für C/S, graue Felder kein signifikanter Unterschied, ${ }^{*}$ primäre Studienvariable ${ }^{* *}$ sekundäre Studienvariable. SF-36 Short Form-36, BP Bodily Pain, VAS visuelle Analogskala, VCSS Venous Clinical Severity Score, HVVSS Homburg Varicose Vein Severity Score, VDS Venous Disability Score, CEAP Clinical-Etiologic-Anatomic-Pathophysiologic Classfication, AVVQ Aberdeen Varicose Veins Questionnaire, EuroQol-5D European Quality of Life - 5 dimensional Questionnaire, CIVIQ2 Chronic Venous Insufficiency Questionnaire 2, nb nicht berichtet 


\section{Methoden}

Auf der Basis einer PubMed-Recherche erfolgte eine selektive Literaturanalyse, konzentriert auf randomisierte kontrollierte Studien (RCT) zum Vergleich von $\mathrm{C} / \mathrm{S}$ und ELT. Berücksichtigt wurden ausschließlich RCTs, die in englischer oder deutscher Sprache verfasst wurden. Folgende Suchbegriffe wurden verwendet: „endovenous laser“, „stripping“, ,varicose veins“. Darüber hinaus wurden weitere Publikationen eingeschlossen, die dem Autor bekannt sind bzw. wenn diese im Rahmen der Literaturzitierungen der gefundenen Studien genannt und als relevant erachtet wurden. Der Suchzeitraum wurde nicht limitiert.

Ausgeschlossen wurden Studien, die beide Verfahren intraindividuell vergleichen (eine Seite C/S, kontralateral ELT). Studien mit C/S versus Krossektomie und ELT wurden ebenfalls nicht berücksichtigt.

Da bereits publizierte systematische Reviews und Metaanalysen, insbesondere das kürzlich von Nesbitt et al. [27] veröffentlichte Update des Cochrane Reviews von 2011, mehrfach auf die eingeschränkte Vergleichbarkeit der Daten hinweisen, ist das Ziel dieser Übersicht der deskriptive Vergleich der relevanten RCTs im Hinblick auf die am häufigsten berichteten Studienvariablen. Hierfür sollen v. a. folgende Aspekte, gewichtet nach ihrer medizinischen Relevanz, dargestellt werden:

- schwerwiegende Komplikationen (Majorkomplikationen),

- langfristige Effektivität - Häufigkeit und klinische Konsequenz bei Auftreten von Rezidiven (Unterscheidung zwischen „echtem“ Krossenrezidiv und Progression der Grunderkrankung),

- longitudinale Dynamik der Krankheitsschwere,

- Nebenwirkungen, Lebensqualität, Patientenzufriedenheit,

- Rekonvaleszenz,

- Behandlungskosten.

\section{Ergebnisse}

Die PubMed-Analyse mit den oben genannten Suchparametern ergab 18 Treffer. Sechs Publikationen wurden ausge- schlossen ( $n=4$ andere Therapieverfahren, $n=1$ intraindividueller Vergleich von $\mathrm{C} / \mathrm{S}$ und ELT, $n=1$ in chinesischer Sprache). Die verbliebenen 12 Publikationen $[2,4,6,7,12,19,33,36-40]$ wurden eingeschlossen und durch weitere 4 Publikationen ergänzt $[5,11,13,19]$. Diese $4 \mathrm{Pu}$ blikationen sind Arbeiten, die weitere Aspekte bzw. Nachbeobachtungszeiten der unter PubMed gefundenen RCTs berichten.

Im Ergebnis wurden 10 Studienkollektive mit insgesamt 2210 eingeschlossenen Patienten in 16 Publikationen identifiziert. Teilweise wurden Studien mit unterschiedlichem Follow-up mehrfach publiziert. Per Protokoll behandelt wurden $n=1057 \mathrm{mit}$ ELT und $n=994 \mathrm{mit} \mathrm{C/S}$. Die Nachbeobachtungszeiten umfassen 2 bis 60 Monate. In den $\bullet$ Abb. 1 und 2 sind die Details dieser Studien dargestellt [2, 4-7, 11-13, 18, 19, 33, 36-40].

\section{Majorkomplikationen}

Es wurden insgesamt 5 tiefe Beinvenenthrombosen nach 2051 Prozeduren diagnostiziert (0,24\%), $n=3$ nach $\mathrm{C} / \mathrm{S}, n=2$ nach ELT. Weitere Majorkomplikationen (Verletzung großer Gefäße oder motorischer Nerven, Lungenembolie, schwer wiegende Infektionen, Tod) wurden nicht berichtet. Die beiden Verfahren sind als gleichermaßen sicher zu betrachten. Eine Besonderheit der ELT sind auftretende Thrombusaszensionen über die saphenofemorale Junktion in die V. femoralis communis. Diese wurden bei 5 Patienten beobachtet $(0,47 \%)$. In allen Fällen trat eine folgenlose Rückbildung nach wenigen Wochen ein, teils spontan, teils unter therapeutischer Antikoagulation.

\section{Langfristige Effektivität}

Die langfristige Effektivität operativer Verfahren zur Beseitigung der Stammvarikose lässt sich, zumindest was das Auftreten klinischer Rezidive anbelangt, die der operierten Krossenregion entspringen, frühestens nach 5 Jahren realistisch einschätzen. Als Surrogatparameter gilt das bereits ab einem Jahr postoperativ auftretende duplexsonographisch detektierbare inguinale Krossenrezidiv (DIR), aus dem sich im weiteren Verlauf klinisch relevante Rezidive entwickeln [9]. Die in den Studien verwendeten Parameter sind für das DIR relativ einheitlich. Für das klinische Rezidiv werden weit überwiegend alle neu auftretenden Varizen gewertet. Die Trennung der Termini „echtes Rezidiv“ - also neu auftretende Varizen im operierten Gebiet - und „Progression der Erkrankung“" wird in den vorliegenden Studien zumeist nicht eindeutig herausgearbeitet.

\section{》) Die in den Studien \\ verwendeten Parameter sind für das DIR relativ einheitlich}

Für lediglich 5 RCTs liegen 2-JahresDaten und für 2 RCTs 5-Jahres-Daten vor (s. $\bullet$ Abb. 1). Die DIR 2 bis 5 Jahre nach ELT werden mit 7-22\% angegeben, nach C/S mit 0-28\%. Im Rahmen der RELACS-Studie konnten wir zeigen, dass unter Verwendung einer Endothelnaht mit Prolene ${ }^{\varpi}$ als Barrieremaßnahme DIR nach $\mathrm{C} / \mathrm{S}$ signifikant seltener auftreten als nach ELT (2 Jahre postoperativ 17,8 vs. $1,3 \%)$. Während dieser Befund mit den Studien von Christenson und Flessenkämper in Einklang steht, haben alle anderen Studien keine signifikanten Unterschiede hinsichtlich der DIR ergeben.

Klinische Rezidive 2 bis 5 Jahre postoperativ treten in allen Studien mit Angaben von 16-47\% nach ELT und 23-55\% nach $\mathrm{C} / \mathrm{S}$ ungeachtet des Ursprungs etwa gleich häufig auf. In nur einer Studie wurde ein signifikanter Unterschied zugunsten der ELT berichtet [5]. Da hingegen 3 RCTs über den Surrogatparameter DIR darauf hinweisen, dass C/S im Vergleich zu ELT im Hinblick auf die Entwicklung klinisch relevanter Krossenrezidive langfristig effektiver sein könnte, bleiben längere Nachbeobachtungszeiträume abzuwarten.

\section{Veränderung der \\ Krankheitsschwere}

In nahezu allen RCTs wurden Scores zur Bewertung der Krankheitsausprägung verwendet, einerseits um die Kollektive präoperativ zu vergleichen, andererseits um anhand der Dynamik der Veränderungen Unterschiede zwischen den 
Therapieverfahren auszumachen. In den meisten Studien wurde der Venous Clinical Severity Score (VCSS) eingesetzt. Im Vergleich von ELT und C/S konnte in keiner Studie ein Unterschied der Krankheitsschwere prä- und postoperativ festgestellt werden. Beide Verfahren sind in der Lage, bis 5 Jahre postoperativ den Ausprägungsgrad der Varizenerkrankung gleichermaßen effektiv zu reduzieren.

\section{Nebenwirkungen, Lebensqualität, Patientenzufriedenheit}

In allen Studien werden Angaben zur Häufigkeit von Nebenwirkungen der folgenden Kategorien gemacht: Infektion, Schmerz, Hämatom, Induration, Dysästhesie, Phlebitis, Dyspigmentierung. Anhand der Aufstellung in $\bullet$ Abb. 2 wird deutlich, dass nach C/S Nebenwirkungen im Vergleich mit ELT häufiger berichtet wurden, insbesondere Infektionen, postoperative Schmerzen und Hämatome. Nach ELT traten häufiger phlebitische Reaktionen und Dyspigmentierungen und in zwei Studien auch stärkere postoperative Schmerzen auf. Bei der Bewertung der Nebenwirkungen sind zwei Aspekte zu berücksichtigen. Zum einen liegen bei der Mehrzahl der berichteten Nebenwirkungen $(69 \%)$ keine Unterschiede zwischen beiden Verfahren vor. Zum anderen wurden in 5/10 RCTs unterschiedliche Anästhesieformen in den Therapiegruppen eingesetzt: C/S überwiegend in Allgemeinnarkose oder Spinalanästhesie, ELT in Tumeszenzlokalanästhesie (TLA). Bei Rasmussen, Pronk und in unserer eigenen Studie wurden alle Prozeduren einheitlich in TLA durchgeführt, bei Flessenkämper in Allgemeinanästhesie. Betrachtet man nur diese Studien mit exakt gleichen intraoperativen Bedingungen, so sind die Nebenwirkungen zwischen beiden Therapiegruppen ausgeglichener, insbesondere das Auftreten postoperativer Schmerzen scheint deutlich mit der Anästhesieform zu korrelieren. Es lässt sich folgern, dass sich postoperative Schmerzen durch die Anwendung der TLA bei C/S so weit reduzieren lassen, dass die ELT sogar eher das ungünstigere Schmerzprofil aufweist.

Alle RCTs konnten einheitlich zeigen, dass die krankheitsbezogene Lebensqualität (AVVQ, CIVIQ) ohne nachweisbare
Unterschiede zwischen beiden Therapieverfahren postoperativ signifikant steigt. Pronk et al. berichteten über Lebensqualitätsvorteile nach C/S. Bei Carradice und in unserer Studie schätzten die Patienten das kosmetische Behandlungsresultat nach ELT günstiger ein, waren aber, wie auch in anderen Studien, mit der Therapie insgesamt gleichermaßen zufrieden.

\section{Rekonvaleszenz}

In neun der randomisierten Studien wurden Daten zur postoperativen Erholung des Patienten erhoben. Untersucht wurde die Wiedererlangung der normalen körperlichen Aktivität und der Arbeitsfähigkeit. In 4/9 Studien wurden hinsichtlich dieser Parameter signifikante Vorteile für die ELT beobachtet, in den restlichen 5 Studien gab es keine Unterschiede zwischen beiden Verfahren. Somit kann resümiert werden, dass die Rekonvaleszenz der Patienten nach ELT schneller verläuft als nach C/S. Der Unterschied zugunsten der ELT beträgt für die Arbeitsfähigkeit über alle Studien, die hierzu Angaben gemacht haben, 4,25 Tage.

\section{Behandlungskosten}

Untersuchungen zum Kostenvergleich zwischen ELT und C/S liegen aktuell nur von 2 Arbeitsgruppen vor. In der Studie von Disselhoff aus den Niederlanden, die das Verfahren der Krossektomie und Kryostripping mit ELT verglich, ließen sich nur geringe Unterschiede bzgl. der Kosteneffektivität beider Verfahren ausmachen, mit der Tendenz etwas geringerer Kosten beim Strippingverfahren. Rasmussen (Dänemark) berichtete in seiner ersten Studie von um ca. 10\% geringeren Behandlungskosten bei der Strippingoperation, während sich diese in seiner zweiten Studie nicht unterschieden.

\section{Diskussion}

Ist nun die endovenöse Lasertherapie wirklich besser als die Stripping-Operation?

US-amerikanische und britische Leitlinien suggerieren deutliche Vorteile für die endovenösen thermoablativen Verfahren, sodass die klassische Operation mit Krossektomie und Stripping als Therapie 2. bzw. 3. Wahl in den Hintergrund tritt $[22,28]$. Bedacht werden sollte hierbei, dass die britische Leitlinie durch das Gesundheitsministerium beauftragt wurde und daher leistungssteuernde Aspekte bei der Bewertung der Studienlage nicht auszuschließen sind. In dem CochraneReview von Nesbitt et al., der im Kern zu den gleichen Ergebnissen kommt wie unsere Analyse, wird darauf hingewiesen, dass die vorliegende Evidenz einen Vorzug für eine der genannten Therapieverfahren nicht erlaubt: „Despite the lack of evidence within our review to support one method of treatment over another, the National Institute for Health and Care Excellence (NICE) published their recommendation in July 2013 that endovenous treatment should constitute the first treatment of choice (...) " [27]. Zu Recht wird auch darauf hingewiesen, dass in den vorliegenden Studien ein Selektionsbias vorliegt, da nur Patienten eingeschlossen wurden, die dem endoluminalen Verfahren zugänglich sind. Daher ist einer Verallgemeinerung wie in der NICE-Guideline äußerst kritisch zu begegnen.

Unsere Untersuchung ist in vielen Aspekten und der Betrachtungsweise des Themas mit dem Cochrane-Review vergleichbar. Allerdings wurden im Gegensatz zu Nesbitt et al. die Arbeiten von Disselhoff und Christenson nicht exkludiert, einerseits, weil bei Disselhoff die Modifikation des Stripping-Manövers mit Kryosonden nicht als Ausschlusskriterium definiert wurde, andererseits, weil die Therapie beider Beine mit unterschiedlichen Verfahren bei 25\% der Patienten bei Christenson zumindest doch Rückschlüsse über das klinische und duplexsonographische Rezidiv erlauben.

Wir haben auf eine Metaanalyse der vorhandenen Daten aufgrund ihrer Heterogenität (Unterschiede u. a. bezüglich Definitionen der Studienvariablen, Nachbeobachtungszeit) zugunsten einer deskriptiven Betrachtung bewusst verzichtet. Bei Nesbitt wurde eine solche für die Parameter „klinisches Rezidiv“, „Rekanalisation“, „Neovaskularisation“ und „technisches Versagen“ durchgeführt. Signifikante Vorteile für die ELT wurden für „Neovaskularisation“ und „technisches Versagen“ ermittelt. Es wurde be- 
reits darauf hingewiesen, dass aufgrund der unterschiedlichen hämodynamischen Situationen postoperativ ein Vergleich von C/S und ELT getrennt nach Neovaskularisation und Rekanalisation nicht sinnvoll ist. Beide Prozesse stellen Ausgangspunkte für ein sich entwickelndes klinisches Rezidiv dar, die als ,duplexsonographisches inguinales Rezidiv" subsumiert und durchaus verglichen werden können. In der Cochrane-Analyse wurde zudem bei den ELT-Patienten der Reflux über Krossenseitenäste nicht als duplexsonographisches Rezidiv berücksichtigt. Der Unterschied im Punkt „technisches Versagen" resultiert aus einem Übertragungsfehler; es wurden das ungünstigere Ergebnis nach ELT in der RELACS-Studie der C/S-Gruppe zugeschrieben.

Evident ist eine Gleichwertigkeit der Sicherheit von ELT und konventioneller Chirurgie, sowie der Effektivität im Hinblick auf die Verbesserung von Krankheitssymptomen und Lebensqualität. Auch die postoperative Nebenwirkungsrate ist bei Anwendung des gleichen Anästhesieverfahrens nicht relevant unterschiedlich. Ein Vorteil für die ELT ist hinsichtlich der Rekonvaleszenz einzuräumen. Beide Verfahren unterscheiden sich, bei aktuell noch unbefriedigender Datenlage, jedoch nicht bezüglich des Auftretens klinisch relevanter Rezidive. Langfristige Analysen, die einen Nachbeobachtungszeitraum von mindestens 5 Jahren aufweisen und die bezüglich des Rezidivursprungs diskriminieren, sind erforderlich, um die für den Patienten wichtige Effektivität beider Verfahren noch besser bewerten zu können.

Das Verfahren mit der größeren Rezidivquote wird langfristig auch höhere Kosten produzieren. Dies ist vor allem deshalb bedeutsam, weil einzelne Studien mit dem Surrogatparameter des duplexsonographischen Rezidivs für die ELT signifikant ungünstigere Ergebnisse aufzeigen.

Signifikante Vorteile für die endovenöse Lasertherapie mit Glasfasern der 1. Generation, die eine Einstufung als Therapie der 1 . Wahl erlauben würden, sind somit anhand der aktuellen Literaturlage unter Berücksichtigung von Studien des höchsten Evidenzlevels nicht abzulesen. Gleiches gilt auch für die neueren thermoab- lativen Verfahren der 2. Generation, da bislang überhaupt nur ein RCT mit einjähriger Nachbeobachtungszeit, zum Vergleich der radiofrequenzinduzierten segmentalen Thermoablation (RSTA, ClosureFast ${ }^{\circledR}$ Methode) mit C/S, vorliegt [38].

\section{》) ELT und konventioneller Chirurgie sind hinsichtlich Sicherheit und Effektivität gleichwertig}

Durch die Etablierung der endovenösen Ablationsverfahren hat die Evidenz in der operativen und interventionellen $\mathrm{Va}$ rizentherapie in den vergangenen 10 Jahren eine für die Phlebologie ganz neue und richtungweisende Qualität gewonnen. Dennoch sind weitere prospektive Studien notwendig, die über ausreichend lange Nachbeobachtungszeiten verfügen, um den Stellenwert der endovenösen Ablationsverfahren gegenüber Krossektomie und Stripping klar einordnen zu können. In diesem Zusammenhang wird zukünftig die Erarbeitung standardisierter Zielparameter für eine bessere Vergleichbarkeit der Studien essenziell sein, um die Evidenz weiter zu erhöhen. Schließlich wäre es bei aktueller Gleichwertigkeit der Verfahren wünschenswert, wenn Kriterien für die Auswahl der Behandlungsmethode im Sinne einer Differenzialindikation vorlägen, um für Patient und Arzt entsprechende therapeutische Sicherheit zu schaffen.

\section{Fazit für die Praxis}

- Die aktive Behandlung der Varikose zielt auf die Vermeidung thrombembolischer Komplikationen und einer sich entwickelnden chronisch-venösen Insuffizienz.

- Krossektomie und Stripping, sowie endovenöse Ablationsverfahren stehen dem Therapeuten zur Verfügung.

- Aktuelle Leitlinien aus den USA und Großbritannien empfehlen die endovenösen thermoablativen Verfahren als Therapie der 1. Wahl.

- Die aktuelle Datenlage aus randomisierten kontrollierten Studien belegt hingegen eine Gleichwertigkeit von
Operation und endovenöser Lasertherapie.

- Langfristige Untersuchungen zur Frage der Rezidivhäufigkeit fehlen weitgehend, ebenso wie vergleichende Untersuchungen der neuesten Ablationstechniken.

- Bei der Krossektomie sind eine qualitativ hochwertige Operation und der Einsatz von Barrieretechniken essenziell, um die Häufigkeit von Rezidiven zu verringern.

- Parameter für die Differenzialindikation „operativ oder endoluminal“, wie z. B. der Venendurchmesser, sollten künftig stärker fokussiert werden.

\section{Korrespondenzadresse}

\section{Dr. K. Rass}

Zentrum für Venen und Haut

Eifelklinik St. Brigida, 52152 Simmerath

knuth.rass@st-brigida.de

\section{Einhaltung ethischer Richtlinien}

Interessenkonflikt. Der Autor erhielt Honorare durch Referententätigkeit für Covidien und Reisekostenunterstützung durch Covidien und Ofa Bamberg.

Dieser Beitrag beinhaltet keine Studien an Menschen oder Tieren.

Open Access Dieser Artikel unterliegt den Bedingungen der Creative Commons Attribution License. Dadurch sind die Nutzung, Verteilung und Reproduktion erlaubt, sofern der/die Originalautor/ en und die Quelle angegeben sind.

\section{Literatur}

1. Almeida Jl, Kaufman J, Göckeritz O, Chopra P, Evans MT, Hoheim DF, Makhoul RG, Richards T, Wenzel C, Raines JK (2009) Radiofrequency endovenous ClosureFAST versus laser ablation for the treatment of great saphenous reflux: a multicenter, single-blinded, randomized study (RECOVERY study). J Vasc Interv Radiol 20(6):752-759

2. Biemans AA, Kockaert $M$, Akkersdijk GP, van den Bos RR, de Maeseneer MG, Cuypers P, Stijnen T, Neumann MH, Nijsten T (2013) Comparing endovenous laser ablation, foam sclerotherapy, and conventional surgery for great saphenous varicose veins. J Vasc Surg 58(3):727-734

3. Brake M, Lim CS, Shepherd AC, Shalhoub J, Davies $\mathrm{AH}$ (2013) Pathogenesis and etiology of recurrent varicose veins. J Vasc Surg 57(3):860-868

4. Carradice D, Mekako Al, Mazari FA, Samuel N, Hatfield J, Chetter IC (2011) Randomized clinical trial of endovenous laser ablation compared with conventional surgery for great saphenous varicose veins. Br J Surg 98(4):501-510 
5. Carradice D, Mekako Al, Mazari FA, Samuel N, Hatfield J, Chetter IC (2011) Clinical and technical outcomes from a randomized clinical trial of endovenous laser ablation compared with conventional surgery for great saphenous varicose veins. $\mathrm{Br} J$ Surg 98(8):1117-1123

6. Christenson JT, Gueddi S, Gemayel G, Bounameaux $\mathrm{H}$ (2010) Prospective randomized trial comparing endovenous laser ablation and surgery for treatment of primary great saphenous varicose veins with a 2-year follow-up. J Vasc Surg 52:1234-1241

7. Darwood RJ, Theivacumar N, Dellagrammaticas D, Mavor Al, Gough MJ (2008) Randomized clinical trial comparing endovenous laser ablation with surgery for the treatment of primary great saphenous varicose veins. Br J Surg 95:294-301

8. De Maeseneer MG, Vandenbroeck CP, Van Schil PE (2004) Silicone patch saphenoplasty to prevent repeat recurrence after surgery to treat recurrent saphenofemoral incompetence: long-term follow-up study. J Vasc Surg 40:98-105

9. De Maeseneer MG, Vandenbroeck CP, Hendriks JM, Lauwers PR, van Schil PE (2005) Accuracy of duplex evaluation one year after varicose vein surgery to predict recurrence at the sapheno-femoral junction after five years. Eur JVasc Endovasc Surg 29:308-312

10. De Maeseneer MG, Philipsen TE, Vandenbroeck CP, Lauwers PR, Hendriks JM, De Hert SG, Van Schil PE (2007) Closure of the cribriform fascia: an efficient anatomical barrier against postoperative neovascularisation at the saphenofemoral junction? A prospective study. Eur J Vasc Endovasc Surg 34(3):361-366

11. Disselhoff BC, der Kinderen DJ, Kelder JC, Moll FL (2008) Randomized clinical trial comparing endovenous laser with cryostripping for great saphenous varicose veins. Br J Surg 95:1232-1238

12. Disselhoff BC, Buskens E, Kelder JC, der Kinderen DJ, Moll FL (2009) Randomised comparison of costs and cost-effectiveness of cryostripping and endovenous laser ablation for varicose veins: 2-year results. Eur J Vasc Endovasc Surg 37(3):357-363

13. Disselhoff BC, der Kinderen DJ, Kelder JC, Moll FL (2011) Five-year results of a randomized clinical trial comparing endovenous laser ablation with cryostripping for great saphenous varicose veins. Br J Surg 98(8):1107-1111

14. Dissemond J (2005) Kompressionstherapie bei Patienten mit Ulcus cruris venosum. Dtsch Arztebl 102(41):A-2788-A-2792

15. Doganci S, Demirkilic U (2010) Comparison of $980 \mathrm{~nm}$ laser and bare-tip fibre with $1470 \mathrm{~nm}$ laser and radial fibre in the treatment of great saphenous vein varicosities: a prospective randomised clinical trial. Eur J Vasc Endovasc Surg 40(2):254-259

16. Eberhardt RT, Reffetto JD (2005) Chronic venous insufficiency. Circulation 111:2398-2409

17. Faubel R, Schäfer I, Augustin M, Bruning G (2010) Long-term results and analyses of correlations 5 years after varicose vein stripping. Phlebologie 39:263-269

18. Flessenkämper I, Hartmann M, Stenger D, Roll S (2013) Endovenous laser ablation with and without high ligation compared with high ligation and stripping in the treatment of great saphenous varicose veins: initial results of a multicentre randomized controlled trial. Phlebology 28(1):16-23

19. Flessenkämper $I H$, Stenger $D$, Hartmann $M$, Hartmann K, Roll S (2015) Zweijahresergebnisse einer prospektiv-randomisierten kontrollierten Multicenterstudie zum Vergleich der offen operativen Therapie vs. der lasergestützten endoluminalen Therapie mit und ohne Crossektomie zur Therapie der Varikose der V. saphena magna. Zentralbl Chir 140(1):27-34
20. Frings N, van-Thann PT, Glowacki P, Subasinghe C (2002) Komplikationen in der Varizenchirurgie und Strategien zu ihrer Vermeidung. Phlebologie 31:26-37

21. Frings $N$, Nelle A, Tran P, Fischer R, Krug W (2004) Reduction of neoreflux after correctly performed ligation of the saphenofemoral junction. A randomized trial. Eur J Vasc Endovasc Surg 28:246-252

22. Gloviczki P, Comerota AJ, Dalsing MC, Eklof BG, Gillespie DL, Gloviczki ML, Lohr JM, McLafferty RB, Meissner MH, Murad MH, Padberg FT, Pappas PJ Passman MA, Raffetto JD, Vasquez MA, Wakefield TW (2011) The care of patients with varicose veins and associated chronic venous diseases: clinical practice guidelines of the Society for Vascular Surgery and the American Venous Forum. J Vasc Surg 53(5 Suppl):2S-48S

23. Goode SD, Chowdhury A, Crockett M, Beech A, Simpson R, Richards T, Braithwaite BD (2010) Laser and radiofrequency ablation study (LARA study): a randomized study comparing radiofrequency ablation an endovenous laser ablation $(810 \mathrm{~nm})$. Eur J Vasc Endovasc Surg 40:246-253

24. Krysa J, Jones GT, van Rij AM (2012) Evidence for a genetic role in varicose veins and chronic venous insufficiency. Phlebology 27(7):329-335

25. Mumme A, Hummel T, Burger $P$, Frings $N$, Hartmann M, Broermann M, Schwahn-Schreiber C, Stenger D, Stücker M (2009) High ligation of the saphenofemoral junction is necessary! Results of the German Groin Recurrence Study. Phlebologie 38:99-102

26. Navarro L, Min RJ, Boné C (2001) Endovenous laser: a new minimally invasive method of treatment for varicose veins-preliminary observations using an $810 \mathrm{~nm}$ diode laser. Dermatol Surg 27:117-122

27. Nesbitt C, Bedenis R, Bhattacharya V, Stansby G (2014) Endovenous ablation (radiofrequency and laser) and foam sclerotherapy versus conventional surgery for great saphenous vein varices. Cochrane Database Syst Rev 7:CD005624

28. NICE clinical guideline 168 (2013) Varicose veins in the legs - the diagnosis and management of varicose veins. http://guidance.nice.org.uk/CG168. Zugegriffen: 27. Februar 2015

29. Nordon IM, Hinchliffe RJ, Brar R, Moxey P, Black SA, Thompson MM, Loftus IM (2011) A prospective double-blind randomized controlled trial of radiofrequency versus laser treatment of the great saphenous vein in patients with varicose veins. Ann Surg 254(6):876-881

30. Pfisterer L, König G, Hecker M, Korff T (2014) Pathogenesis of varicose veins - lessons from biomechanics. Vasa 43(2):88-99

31. Pröbstle TM, Lehr HA, Kargl A, Espinola-Klein C, Rother W, Bethge S, Knop J (2002) Endovenous treatment of the greater saphenous vein with a 940nm diode laser: thrombotic occlusion after endoluminal thermal damage by laser-generated steam bubbles. J Vasc Surg 35:729-736

32. Pröbstle TM, Moehler T, Gül D, Herdemann S (2005) Endovenous treatment of the great saphenous vein using a 1,320 nm Nd:YAG laser causes fewer side effects than using a $940 \mathrm{~nm}$ diode laser. Dermatol Surg 31(12):1678-1683

33. Pronk P, Gauw SA, Mooij MC, Gaastra MT, Lawson JA, van Goethem AR, van Vlijmen-van Keulen CJ (2010) Randomised controlled trial comparing sapheno-femoral ligation and stripping of the great saphenous vein with endovenous laser ablation (980 $\mathrm{nm}$ ) using local tumescent anaesthesia: one year results. Eur J Vasc Endovasc Surg 40:649-656
34. Rabe E, Pannier-Fischer F, Bromen $\mathrm{K}$, Schuldt $\mathrm{K}$, Stang A, Poncar C, Wittenhorst M, Bock E, Weber S, Jöckel KH (2003) Bonn Vein Study by the German Society of Phlebology. Epidemiological study to investigate the prevalence and severity of chronic venous disorders in the urban and rural residential populations. Phlebologie 32:1-14

35. Rafetto JD (2009) Dermal pathology, cellular biology, and inflammation in chronic venous disease. Thromb Res 123(Suppl 4):S66-S71

36. Rasmussen $L H$, Bjoern L, Lawaetz $M$, Blemings $A$, Lawaetz B, Eklof B (2007) Randomized trial comparing endovenous laser ablation of the great saphenous vein with high ligation and stripping in patients with varicose veins: short-term results. JVasc Surg 46(2):308-315

37. Rasmussen $L H$, Bjoern L, Lawaetz M, Lawaetz $B$, Blemings A, Eklöf B (2010) Randomised clinical trial comparing endovenous laser ablation with stripping of the great saphenous vein: clinical outcome and recurrence after 2 years. Eur JVasc Endovasc Surg 39:630-635

38. Rasmussen LH, Lawaetz M, Bjoern L, Vennits B, Blemings A, Eklof B (2011) Randomized clinical trial comparing endovenous laser ablation, radiofrequency ablation, foam sclerotherapy and surgical stripping for great saphenous varicose veins. $\mathrm{Br} J$ Surg 98(8):1079-1087

39. Rasmussen L, Lawaetz M, Bjoern L, Blemings A, Eklof $B$ (2013) Randomized clinical trial comparing endovenous laser ablation and stripping of the great saphenous vein with clinical and duplex outcome after 5 years. J Vasc Surg 58(2):421-426

40. Rass K, Frings N, Glowacki P, Hamsch C, Gräber S, Vogt T, Tilgen W (2012) Comparable effectiveness of endovenous laser ablation and high ligation with stripping of the great saphenous vein - twoyear results of a randomized clinical trial (RELACS study). Arch Dermatol 148(1):49-58

41. van Rij AM, Jones GT, Hill BG, Amer M, Thomson IA, Pettigrew RA, Packer SG (2008) Mechanical inhibition of angiogenesis at the saphenofemoral junction in the surgical treatment of varicose veins: early results of a blinded randomized controlled trial. Circulation 118(1):66-74

42. Schellhaaß A, Walther A, Konstantinides S, Böttiger BW (2010) Diagnostik und Therapie bei akuter Lungenembolie. Dtsch Arztebl Int 107(3435):589-595

43. Shepherd AC, Gohel MS, Brown LC, Metcalfe MJ, Hamish M, Davies AH (2010) Randomized clinical trial of VNUS ${ }^{\circledR}$ ClosureFast $^{\mathrm{TM}}$ radiofrequency ablation versus laser for varicose veins. Br J Surg 97:810818

44. Theivacumar NS, Dellagrammaticas D, Beale RJ, Mavor AIS, Gough MJ (2008) Factors influencing the effectiveness of endovenous laser ablation (EVLA) in the treatment of great saphenous vein reflux. Eur J Vasc Endovasc Surg 35:119-123

45. Theivacumar NS, Darwood R, Gough MJ (2009) Neovascularisation and recurrence 2 years after varicose vein treatment for sapheno-femoral and great saphenous vein reflux: a comparison of surgery and endovenous laser ablation. Eur JVasc Endovasc Surg 38:203-207

46. Weiss RA (2002) Comparison of endovenous radiofrequency versus $810 \mathrm{~nm}$ diode laser occlusion of large veins in an animal model. Dermatol Surg 28:56-61 